\title{
Pre- and Post-Processing for Optimal Noise Reduction in Cyclic Prefix Based Channel Equalizers
}

\author{
Bojan Vrcelj and P. P. Vaidyanathan \\ Dept. of Electrical Engineering 136-93 \\ California Institute of Technology \\ Pasadena, CA 91125-0001
}

\begin{abstract}
Cyclic prefix based equalizers are widely used for high-speed data transmission over frequency selective channels. Their use in conjunction with DFT filterbanks is especially attractive, given the low complexity of implementation. Some examples include the DFT-based DMT systems. In this paper we consider a general cyclic prefix based system for communication and show that the equalization performance can be improved by simple preand post-processing aimed at reducing the noise at the receiver. This processing is done independently of the ISI cancellation performed by the frequency domain equalizer. ${ }^{1}$
\end{abstract}

\section{INTRODUCTION}

There has been considerable interest in applying the equalization techniques based on cyclic prefix to high speed data transmission over frequency selective channels, such as twisted pair channels in telephone cables [1], [2]. Cyclic prefix systems are invariably used in conjunction with the discrete Fourier transform (DFT) matrices, and one example is the DFT-based discrete multitone modulation (DMT) system [3]. Although it has been shown by Kalet [9] that the use of ideal transmit and receive filters in the multitone environment can lead to achieving signal to noise ratio (SNR) within 8-9 dB of the channel capacity (depending on the probability of error), in practice the DFT filterbank is most commonly used because of its low complexity. The development of these systems lead, for example, to asymmetric digital subscriber loops (ADSL) and high-speed digital subscriber loops (HDSL) [2], [3].

The initial goal of cyclic prefix based methods for equalization is to cancel the intersymbol interference (ISI) induced by the channel. This task is achieved by introducing some redundancy in the form of a cyclic prefix (see Sec. II). Depending on the nulls of the channel in question, the noise can get severely amplified by the equalizer alone. In this paper we consider a general cyclic prefix based system with the aim of further reducing the noise at the receiver. This is achieved by performing the ISI cancellation and the noise suppression separately, in different modules. The main module for ISI cancellation is based on cyclic prefix with DFT filterbanks and is unchanged.

\footnotetext{
${ }^{1}$ Work supported in part by the ONR grant N00014-99-1-1002, USA.
}

It is preceded (followed) by the optimal precoder (equalizer) for the given input and noise statistics. These blocks are realized by constant matrix multiplication, so that the overall communications system remains of low complexity.

In the following we first give a brief overview of the cyclic prefix system with DFT matrices used as the basic ISI canceler. Then, we introduce a way to deal with noise suppression separately and derive the optimal constrained pair precoder/equalizer for this purpose. The constraint is that in the absence of noise the overall system is still ISI-free. The performance of the proposed method is evaluated through computer simulations and a significant improvement with respect to the original system without pre- and post-processing is demonstrated.

Throughout this paper we assume that the frequency selective channel is a known FIR system of order $L$, i.e.

$$
C(z)=\sum_{k=0}^{L} c(k) z^{-k} .
$$

The notations $\mathbf{A}^{T}$ and $\mathbf{A}^{\dagger}$ represent the transpose and transpose conjugate of a matrix $\mathbf{A}$.

\section{CYCLIC PREFIX SYSTEM WITH DFT MATRICES}

In this section we give a review of a cyclic prefix based system for channel equalization that employs DFT filterbanks. Before we demonstrate the complete system, we first introduce the concept of cyclic prefix. This is a way of inserting redundancy into the input data stream and it becomes useful in the process of channel equalization as will be explained later. Consider the symbol stream from Fig. 1(a). It is divided into blocks of length $M$. The last $L$ symbols from each block are copied and inserted at the beginning of that block (we assume hereafter that the redundancy $L<M)$. This is achieved by "squeezing" the samples as explained in Fig. 1(b)-(c). Obviously, this operation of inserting the redundancy into the input data stream results in the bandwidth expansion. In this case the bandwidth expansion ratio is given by $\alpha=(M+L) / M$, which can be made sufficiently small by making $M$ large. The purpose of 
(a)

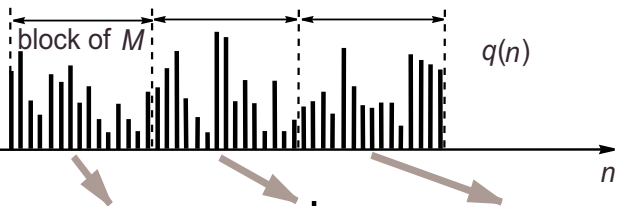

(b)

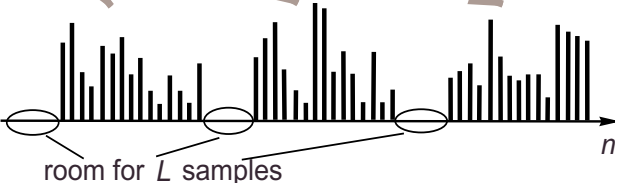

(c)

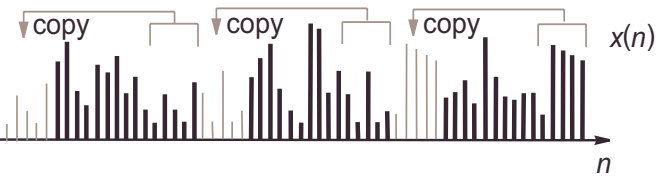

Figure 1: (a) Input symbol stream, (b)-(c) explanation of how cyclic prefix is inserted.

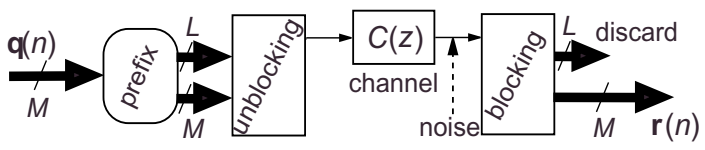

Figure 2: Channel with the system for cyclic prefix.

inserting the cyclic prefix is to allow the receiver to remove ISI using some low-complexity operations.

As the first step in understanding how this is achieved, consider Fig. 2, ignoring the noise for the time being. The box labeled "blocking" is a serial to parallel converter, similarly "unblocking" performs the reverse operation and the block "prefix" inserts the cyclic prefix as described in Fig. 1. Notice that the blocked sequences $\mathbf{q}(n)$ and $\mathbf{r}(n)$ are related through a multiplication by the $M \times M$ right circulant [5] channel matrix C. The first column of $\mathbf{C}$ consists of the $L+1$ coefficients of the channel impulse response $c(k)$ followed by $M-L-1$ zeros. Since we can safely assume ${ }^{2} c(0) \neq 0$, it follows that $\mathbf{C}$ is nonsingular and the effect of the channel can be neutralized by inverting it. Next, note that any circulant matrix can be diagonalized by a DFT matrix [5]. Thus we can write [5]

$$
\mathbf{C}=\mathbf{W}^{-1} \boldsymbol{\Gamma}_{\mathbf{e}} \mathbf{W}
$$

where $\mathbf{W}$ is the $M \times M$ DFT matrix and the diagonal matrix $\boldsymbol{\Gamma}_{\mathrm{e}}$ has the eigenvalues of $\mathbf{C}$ on its diagonal. Those eigenvalues, in turn, are nothing but the $M$-point DFT coefficients of $c(k)$,

\footnotetext{
${ }^{2}$ Otherwise we can shorten the impulse response of $C(z)$.
}

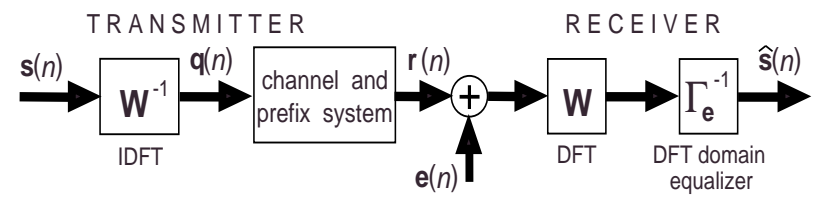

Figure 3: Conventional cyclic prefix system with DFT matrices used for ISI cancellation.

thus we have

$$
\boldsymbol{\Gamma}_{\mathbf{e}}=\left[\begin{array}{ccccc}
C_{M}[0] & 0 & 0 & \ldots & 0 \\
0 & C_{M}[1] & 0 & \ldots & 0 \\
\vdots & & \ddots & \vdots & \\
0 & 0 & 0 & \ldots & C_{M}[M-1]
\end{array}\right]
$$

with $C_{M}[n]=\sum_{k=0}^{L} c(k) e^{-j 2 \pi k n / M}$. As we noted before, the ISI cancellation is achieved by inverting $\mathbf{C}$ in (1). Therefore, after moving the matrix $\mathbf{W}^{-1}$ to the transmitter side, the complete DFT-based cyclic prefix system is shown in Fig. 3. The block labeled "channel and prefix system" is given in Fig. 2. Matrix $\Gamma_{\mathbf{e}}{ }^{-1}$ is better known as the frequency domain equalizer and contains on its diagonal the reciprocals of the DFT coefficients of the channel. The input vector sequence $\mathbf{s}(n)$ in Fig. 3 is obtained by blocking the symbol stream into blocks of length $M$. The noise vector $\mathbf{e}(n)$ is obtained by blocking the physical noise process into blocks of length $M+L$ and then discarding $L$ samples out of each block (see Fig. 2).

The main merit of the cyclic prefix system with DFT matrices lies in its simplicity. From Fig. 3 we see that the transmitter only needs to perform the inverse DFT operation followed by the cyclic prefix insertion. On the other hand, the receiver removes the cyclic prefix, performs the DFT operation and frequency domain equalization, which amounts simply to multiplying each channel by a constant. Moreover, if $M$ is chosen as a power of two, both IDFT and DFT operations can be implemented using the fast radix- 2 algorithm. The noise vector process $\mathbf{e}(n)$ at the receiver passes through the DFT matrix $\mathbf{W}$ and the frequency domain equalizer $\boldsymbol{\Gamma}_{\mathbf{e}}{ }^{-1}$. Since $\mathbf{W}$ is a scaled unitary matrix, the main contribution to amplifying the noise power at the receiver comes from the multipliers $1 / C_{M}[n]$ embedded in $\boldsymbol{\Gamma}_{\mathbf{e}}{ }^{-1}$. Obviously, if the channel $C(z)$ has zeros near the unit circle, these multipliers can get large, consequently boosting the noise at the receiver and degrading the performance. In the next section we propose a simple modification of the basic equalization structure from Fig. 3 that will improve the performance significantly.

\section{MODIFIED SYSTEM FOR NOISE SUPPRESSION}

Consider the system shown in Fig. 4(a)-(b). It consists of the same basic cyclic prefix transceiver (the middle portion) 

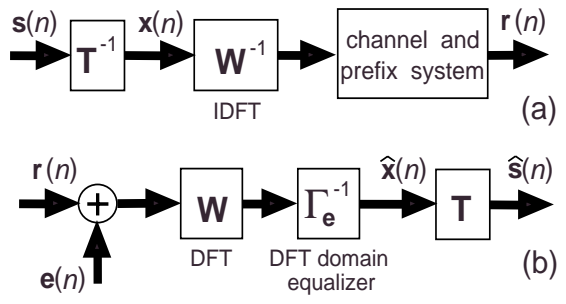

Figure 4: Cyclic prefix system with separated ISI cancellation and noise suppression.

surrounded by the constant matrices $\mathbf{T}^{-1}$ and $\mathbf{T}$, so that the transmitter of the new system is shown in Fig. 4(a), while the receiver is in Fig. 4(b). Notice that as for the signal part, this system is completely equivalent to the one from Sec. II, i.e. the signal still goes through an identity system. Therefore, the only purpose of the precoder $\mathbf{T}^{-1}$ and the corresponding equalizer $\mathbf{T}$ is to reduce the noise power at the receiver output $\hat{\mathbf{s}}(n)$. It is clear that without any additional constraints this task can be trivially accomplished by scaling the matrix $\mathbf{T}$ by a very small constant; without changing the received signal, in this way we could arbitrarily reduce the received noise power. However, this would in turn arbitrarily increase the power in the transmitted signal $\mathbf{x}(n)$, which is of course unacceptable. The remedy, therefore, is to find the optimal precoder/equalizer pair subject to the constraint on the transmitted power. This constraint can be written as

$$
\operatorname{Tr}\left\{E\left[\mathbf{x} \mathbf{x}^{\dagger}\right]\right\}=\operatorname{Tr}\left\{\mathbf{T}^{-1} \mathbf{R}_{s s}\left(\mathbf{T}^{-1}\right)^{\dagger}\right\} \leq P_{x},
$$

where $\operatorname{Tr}\{\cdot\}$ and $E[\cdot]$ denote the trace and expectation operators respectively and $\mathbf{R}_{s s}$ denotes the autocorrelation matrix of the input vector process $\mathbf{s}(n)$. Quantity $P_{x}$ denotes the maximum power in the transmit signal $\mathbf{x}(n)$. From now on, we make the assumption that the input symbols are independent, identically distributed, coming from a predefined constellation. Therefore, the autocorrelation matrix becomes $\mathbf{R}_{s s}=P_{s} \mathbf{I}_{M}$, with $\mathbf{I}_{M}$ denoting the $M \times M$ identity matrix, and $P_{s}$ the power in the input symbol stream. Thus, if the transmit power needs to be unchanged after employing the precoder, the power constraint becomes

$$
\operatorname{Tr}\left\{\mathbf{T}^{-1}\left(\mathbf{T}^{-1}\right)^{\dagger}\right\}=\operatorname{Tr}\left\{\left(\mathbf{T}^{-1}\right)^{\dagger} \mathbf{T}^{-1}\right\}=1
$$

using the identity $\operatorname{Tr}\{\mathbf{A B}\}=\operatorname{Tr}\{\mathbf{B} \mathbf{A}\}$, for $\mathbf{A}, \mathbf{B}$ square.

According to the polar decomposition theorem [7] an arbitrary square matrix $\mathbf{T}$ can be written as a product $\mathbf{T}=\mathbf{U P}$, where $\mathbf{U}$ is unitary and $\mathbf{P}$ a positive semidefinite matrix (in our case, since $\mathbf{T}$ is obviously desired to be nonsingular, $\mathbf{P}$ is positive definite). Therefore, the power constraint (4) becomes

$$
\operatorname{Tr}\left\{\mathbf{U}^{\dagger} \mathbf{U}\left(\mathbf{P}^{-1}\right)^{\dagger} \mathbf{P}^{-1}\right\}=\operatorname{Tr}\left\{\mathbf{P}^{-2}\right\}=1 .
$$

Our objective as mentioned before is to minimize the output noise power (subject to the power constraint). This can be written as

$$
\begin{aligned}
& \min _{\mathbf{T}} \operatorname{Tr}\left\{\mathbf{T} \boldsymbol{\Gamma}_{\mathbf{e}}{ }^{-1} \mathbf{W} \mathbf{R}_{e e} \mathbf{W}^{\dagger}\left(\boldsymbol{\Gamma}_{\mathbf{e}}{ }^{-1}\right)^{\dagger} \mathbf{T}^{\dagger}\right\}, \text { that is } \\
& \min _{\mathbf{P}} \operatorname{Tr}\left\{\mathbf{P}^{\dagger} \mathbf{P Q}\right\}, \quad \mathbf{Q} \triangleq \boldsymbol{\Gamma}_{\mathbf{e}}{ }^{-1} \mathbf{W} \mathbf{R}_{e e} \mathbf{W}^{\dagger}\left(\boldsymbol{\Gamma}_{\mathbf{e}}{ }^{-1}\right)^{\dagger}
\end{aligned}
$$

Notice that neither the objective (6) nor the constraint (5) depend on the choice of the unitary matrix $\mathbf{U}$. This leads us to conclude that without loss of generality we can choose $\mathbf{U}=$ $\mathbf{I}_{M}$, or $\mathbf{T}=\mathbf{P}$. Furthermore, it follows that we can achieve nothing in terms of noise suppression if $\mathbf{T}$ itself is unitary [this can also be concluded directly from Fig. 4(b)].

In order to solve this problem of nonlinear optimization, namely minimizing (6) subject to (5), it is useful to consider the unitary diagonalization of the positive definite matrices

$$
\mathbf{P}=\mathbf{U}_{p} \boldsymbol{\Lambda}_{p} \mathbf{U}_{p}^{\dagger}, \quad \mathbf{Q}=\mathbf{U}_{q} \boldsymbol{\Lambda}_{q} \mathbf{U}_{q}^{\dagger} .
$$

Defining the unitary matrix $\mathbf{V} \triangleq \mathbf{U}_{q}^{\dagger} \mathbf{U}_{p}$, the objective function can be written as

$$
\min _{\mathbf{V}, \boldsymbol{\Lambda}_{p}} \operatorname{Tr}\left\{\mathbf{V}^{\dagger} \boldsymbol{\Lambda}_{q} \mathbf{V} \boldsymbol{\Lambda}_{p}^{2}\right\}
$$

Notice that the matrices $\mathbf{V}$ and $\boldsymbol{\Lambda}_{p}$ are arbitrary, subject to being unitary and diagonal positive definite, respectively. More importantly, optimal $\boldsymbol{\Lambda}_{p}$ can be chosen independently of optimal V. Let us denote

$$
\mathbf{A} \triangleq \mathbf{V}^{\dagger} \boldsymbol{\Lambda}_{q} \mathbf{V}
$$

and the diagonal elements of $\mathbf{A}$ by $a_{1}, a_{2}, \ldots, a_{M}$. Also denote the diagonal elements of $\boldsymbol{\Lambda}_{p}$ by $\lambda_{p, 1}, \lambda_{p, 2}, \ldots, \lambda_{p, M}$ and the diagonal elements of $\boldsymbol{\Lambda}_{q}$ by $\lambda_{q, 1}, \lambda_{q, 2}, \ldots, \lambda_{q, M}$. When considered as a function of $\left\{\lambda_{p, i}\right\}$, the minimization problem becomes

$$
\min _{\lambda_{p, i}} \sum_{i=1}^{M} a_{i} \lambda_{p, i}^{2}, \quad \text { s.t. } \sum_{i=1}^{M} \lambda_{p, i}^{-2}=1 .
$$

The problem nicely reduces to scalar optimization and this can be readily solved using the method of Lagrange multipliers. The optimal solution for $\left\{\lambda_{p, i}\right\}$ as a function of the diagonal elements of $\mathbf{A}$ is given by

$$
\lambda_{p, i}^{(o p t)}=\left(\frac{\sqrt{a_{i}}}{\sum_{i=1}^{M} \sqrt{a_{i}}}\right)^{-1 / 2} .
$$

The next step is to find the optimal set of diagonal elements of $\mathbf{A}$, namely $\left\{a_{i}\right\}$ that minimize the objective (10). After substituting the solution for $\left\{\lambda_{p, i}\right\}$, the problem becomes

$$
\min _{a_{i}}\left[\sum_{i=1}^{M} a_{i}+\sum_{i \neq j} \sqrt{a_{i} a_{j}}\right]
$$




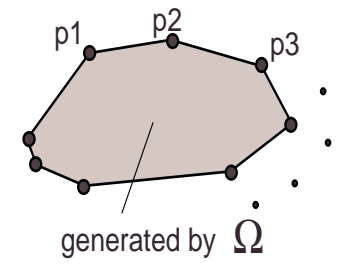

Figure 5: Convex polytope defined by the doubly stochastic matrix $\Omega$; see text.

Notice from (9) that $\sum_{i=1}^{M} a_{i}=\operatorname{Tr}\left\{\boldsymbol{\Lambda}_{q}\right\}=$ const. (since $\mathbf{Q}$ is fixed), so that the objective given by (12) becomes that of minimizing $f(\mathbf{a}) \triangleq \sum_{i \neq j} \sqrt{a_{i} a_{j}}$, with the vector a defined as $\mathbf{a} \triangleq\left[a_{1}, a_{2}, \ldots, a_{M}\right]^{T}$. It is important here to notice that $f(\cdot)$ is a concave function of $\mathbf{a}$, since it is a positive linear combination of concave functions of the form $\sqrt{a_{i} a_{j}}$. If we denote by $v_{i, j}$ the $(i, j)$ th element of $\mathbf{V}$ defined previously, then from (9) we have

$$
a_{i}=\sum_{k=1}^{M}\left|v_{k, i}\right|^{2} \lambda_{q, k}
$$

or in other words

$$
\mathbf{a}=\boldsymbol{\Omega} \times\left[\lambda_{q, 1} \lambda_{q, 2} \ldots \lambda_{q, M}\right]^{T} .
$$

Here $\boldsymbol{\Omega}$ is a doubly stochastic matrix [7], with the $(i, j)$ th element given by $\boldsymbol{\Omega}_{i, j} \triangleq\left|v_{j, i}\right|^{2}$. It follows [8] that (13) defines a convex polytope in the first quadrant of the real $M$ dimensional vector space. This is shown in Fig. 5. The corners of that polytope are given by the permutations of the vector $\left[\lambda_{q, 1}, \lambda_{q, 2}, \ldots, \lambda_{q, M}\right]^{T}$ and are denoted by $p_{i}$ in Fig. 5. Since $f(\cdot)$ is a concave function defined over a convex polytope its absolute minimum is reached at one of these corners. That is to say, the optimal matrix $\mathbf{V}$ is a permutation matrix. But from the form of the objective function $f(\mathbf{a})$ we conclude that without loss of generality we can take $\mathbf{V}=\mathbf{I}_{M}$. Summarizing, we have shown the following.

Theorem. Optimal precoder/equalizer. Consider the system for digital communication shown in Fig. 4. The optimal precoder/equalizer matrix $\mathbf{T}$ in the sense of minimizing the output noise power for the fixed transmitted power constrained as in (3) is given by $\mathbf{T}=\mathbf{U}_{q} \boldsymbol{\Lambda}_{p} \mathbf{U}_{q}^{\dagger}$. Unitary matrix $\mathbf{U}_{q}$ is obtained as in (7), given the definition of $\mathbf{Q}$ in (6). The diagonal elements of $\boldsymbol{\Lambda}_{p}$ are given by

$$
\lambda_{p, i}^{(o p t)}=\left(\frac{\sqrt{\lambda_{q, i}}}{\sum_{i=1}^{M} \sqrt{\lambda_{q, i}}}\right)^{-1 / 2} .
$$

\section{EXPERIMENTAL RESULTS}

We now consider an experimental example designed to compare the equalization in a traditional cyclic prefix system versus the modified system with optimal precoder for noise suppression. The channel $C(z)$ was taken to be of order $L=8$, with coefficients $1.0,-0.5974,0.2346,-0.0305,0.8519,0.5680$, $0.0975,-1.0479,0.6939$. The magnitudes of the four complex pairs of zeros of $C(z)$ are given by

$$
0.6839,0.9656,1.0793,1.1687 \text {. }
$$

We see that four complex zeros of $C(z)$ are very close to the unit circle. As a consequence, several DFT coefficients of $C_{M}[n]$ are very low in magnitude, so that the frequency domain equalizer at those frequencies amplifies the noise severely. This resulted in very high probabilities of error at signal to noise ratios that are moderate to high, when the traditional cyclic prefix system is used. This can be seen from Fig. 8. The quantity $M$ was chosen to be 128, so that the FFT algorithm can be used. The input constellation was chosen to be 64-QAM. The signal to noise ratio used in the experiments was calculated at the input of the receiver [see Fig. 4(b)], i.e.

$$
\mathrm{SNR}=20 \log _{10} \frac{\|\mathbf{r}\|_{2}}{\|\mathbf{e}\|_{2}} .
$$

In Figs. 6-7 we show the results of the channel equalization in the traditional system and the modified system with precoder, respectively. The plots are obtained at the signal to noise ratio of $24 \mathrm{~dB}$. The average probability of error corresponding to the traditional system from Fig. 6 is slightly more than $8 \times 10^{-3}$, while the error probability in the system with precoder is less than $2 \times 10^{-5}$. In Fig. 8 we show the average symbol error probability as a function of signal to noise ratio for the two systems (traditional and with pre- and postprocessing). We see that in this example even at the moderate probabilities of error the improvement is significant (more than 6-8 dB).

\section{CONCLUDING REMARKS}

We have considered a cyclic prefix system with DFT matrices, that is commonly used in several equalization methods, such as the DFT-based DMT. Note that apart from the frequency domain equalizer introduced in Sec. II, in practice there would typically be a time domain equalizer for channel shortening as well [6], but this was not considered here. Our approach was to treat the problem of noise suppression separately from the ISI cancellation and implement it as a separate module. With this in mind we have constructed the optimal constant (precoder, equalizer) pair for noise reduction, subject to the constraint that there is no ISI in the absence of noise. Obviously, a more general solution would involve matrices with memory and/or substitute the restraint on the ISI-free solution 


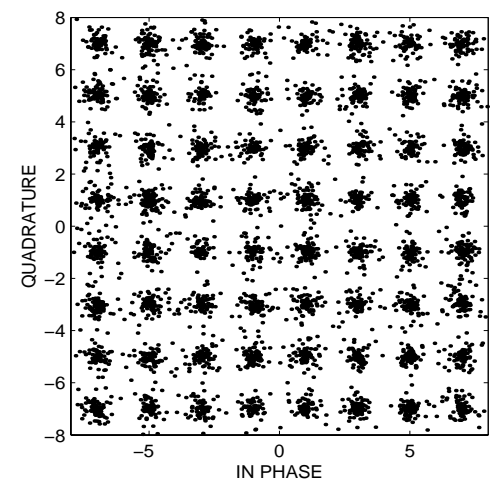

Figure 6: Equalization results in a traditional system without optimal pre- and post-processing.

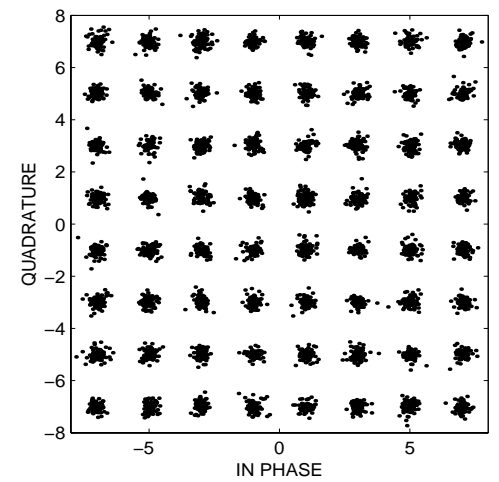

Figure 7: Equalization results using a modified system with optimal precoder/equalizer.

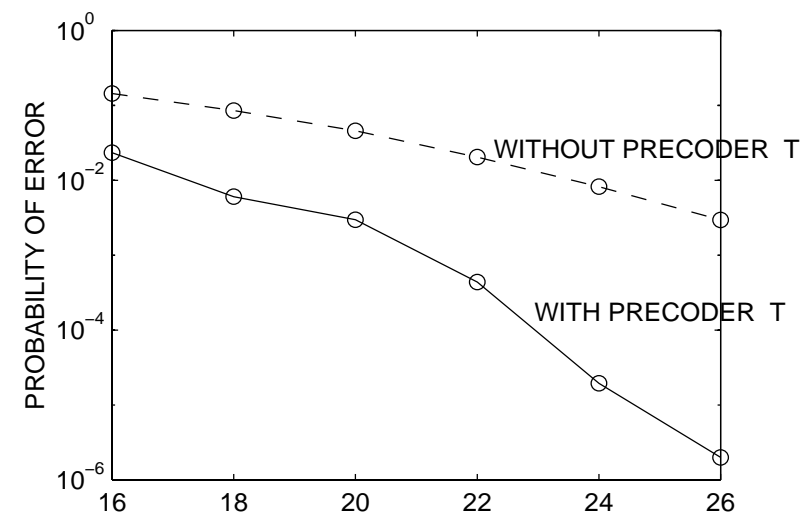

Figure 8: Probability of error vs. SNR: without precoder (dashed line) and with precoder (solid line). by the minimum mean-squared error objective. A similar approach was taken in [10], although the authors there do not consider a cyclic prefix system, and unfortunately the solution in that case involves ideal (unrealizable) filtering. Another generalization of our approach would involve a "tall" instead of a square matrix precoder, and thus allow for some additional redundancy in the system. Moreover, a similar approach could be applied to modified DFT-based systems and generalized perfect DMT systems [4]. Finally, note that although our initial goal was not to allocate power in subbands, but perform a more general precoding and equalization for noise reduction, some sort of power allocation is performed here as well. In particular, in the case when the noise autocorrelation matrix is a scaled identity, one can easily show that the optimal matrix $\mathbf{T}$ becomes indeed diagonal. Thus, in this case the optimal strategy is to allocate as much signal power in the frequency bands where the channel magnitude is low, so that the equalizer at those frequencies does not amplify the noise.

\section{REFERENCES}

[1] J. A. C. Bingham, "Multicarrier modulation for data transmission: an idea whose time has come," IEEE Comm. Magazine, pp. 5-14, May 1990.

[2] T. Starr, J. M. Cioffi, P. J. Silverman, Understanding DSL technology. Prentice Hall, Inc., 1999.

[3] P. S. Chow, J. C. Tu and J. M. Cioffi, "Performance evaluation of a multichannel transceiver system for ADSL and VHDSL services," IEEE J. Select. Areas Comm., vol. 9, pp. 909-919, Aug. 1991.

[4] Y.-P. Lin and S.-M. Phoong, "Perfect discrete multitone modulation with optimal transceivers," IEEE Trans. Signal Proc., vol. 48(6), pp. 1702-1711, Jun. 2000.

[5] A. Papoulis, Signal Analysis. McGraw Hill, 1977.

[6] J. G. Proakis, Digital Communications, McGraw Hill, Inc., 1995.

[7] R. A. Horn and C. R. Johnson, Matrix Analysis. Cambridge Univ. Press, Cambridge, UK, 1985.

[8] P. P. Vaidyanathan and S. Akkarakaran, "A review of the theory and applications of optimal subband and transform coders," Appl. and Computat. Harm. Anal., vol. 10, pp. 254-289, 2001.

[9] I. Kalet, "The multitone channel," IEEE Trans. Communications, vol. 37, pp. 119-124, Feb. 1989.

[10] J. Yang and S. Roy, "On joint transmitter and receiver optimization for multiple-input-multiple-output (MIMO) transmission systems," IEEE Trans. Comm., vol. 42(12), pp. 3221-3231, Dec. 1994. 